\title{
ASO Visual Abstract: Liver Stiffness Measured by Two- Dimensional Shear Wave Elastography for Predicting Symptomatic Post-Hepatectomy Liver Failure in Patients with Hepatocellular Carcinoma
}

Haiyi Long, MD, Xian Zhong, MD, Liya Su, MD, Tongyi Huang, MD, Yu Duan, MD, Weiping Ke, BE, Xiaoyan Xie, MD, and Manxia Lin, MD

Department of Medical Ultrasonics, Institute of Diagnostic and Interventional Ultrasound, The First Affiliated Hospital of Sun Yat-sen University, Guangzhou, China

Liver stiffness measured using two-dimensional shear wave elastography could predict symptomatic post-hepatectomy liver failure using the best cut-off value of $9.5 \mathrm{kPa}$.

Child-Turcotte-Pugh grade was a stronger indicator of symptomatic post-hepatectomy liver failure in minor hepatectomy, while liver stiffness was more effective in selecting candidates for major hepatectomy.
Liver stiffness could further stratify the risk of symptomatic post-hepatectomy liver failure in Child-TurcottePugh grade A patients, regardless of the extent of hepatectomy (https://doi.org/10.1245/s10434-021-10563-4).

Supplementary Information The online version contains supplementary material available at https://doi.org/10.1245/s10434021-10674-y.

Publisher's Note Springer Nature remains neutral with regard to jurisdictional claims in published maps and institutional affiliations.

(C) Society of Surgical Oncology 2021

Published Online: 14 September 2021

X. Xie, MD

e-mail: xiexyan@mail.sysu.edu.cn

M. Lin, MD

e-mail: linmxia@mail.sysu.edu.cn 